(2) Open Access Full Text Article

\title{
A case report of toxic epidermal necrolysis associated with AZD-929I
}

This article was published in the following Dove Press journal:

Drug Design, Development and Therapy

\section{Jie Wang \\ XianYe Cheng \\ Yan Lu \\ BingRong Zhou}

Department of Dermatology, The First Affiliated Hospital of Nanjing Medical University, Nanjing, Jiangsu, The People's Republic of China

Correspondence: BingRong Zhou Department of Dermatology, The First Affiliated Hospital of Nanjing Medical University, 300 Guangzhou Road, Nanjing, Jiangsu 210029, The People's

Republic of China

Tel +86 I358402 0I 23

Fax +86 2087257353

Email bingrong.2002@163.com

\begin{abstract}
Epidermal growth factor receptor-tyrosine kinase inhibitors (EGFR-TKIs) are a strain of small molecule inhibitors mainly used to treat the metastatic non-small cell lung cancer. Their predominant adverse effect is skin toxicity, usually manifested as acneiform rash, skin fissure, xerosis, and paronychia. Severe epidermal necrosis and exfoliation rarely occur. As one of the new generation of epidermal growth factor receptor-tyrosine kinase inhibitors, AZD-9291 is claimed to have better efficacy and fewer side effects, particularly appropriate for patients with EGFR T790M mutation. Herein we report a 51-year-old man who developed a large area of skin necrosis and was diagnosed with toxic epidermal necrolysis after AZD-9291 ingestion.
\end{abstract}

Keywords: TEN, AZD-9291, osimertinib, EGFR-TKIs, NSCLC

\section{Introduction}

Toxic epidermal necrolysis (TEN) is a life-threatening skin reaction characterized by mucocutaneous blistering and sloughing accompanied by sudden onset of high fever and signs of systemic toxicity. It is classified into the spectrum of diseases with Stevens-Johnson syndrome (SJS), differing from each other in the level of epidermal detachment. SJS is diagnosed when the epidermal detachment involves $<10 \%$ of the body surface area, TEN when $>30 \%$ is involved, and SJS/TEN overlap when $10 \%-30 \%$ is involved. ${ }^{1}$ Though the typical clinical manifestation of SJS/TEN makes it easy to be diagnosed, biopsy and detailed medical history collection are of great help.

As a strain of small molecular inhibitors, epidermal growth factor receptortyrosine kinase inhibitors (EGFR-TKIs) have been established as a first-line therapy for patients with advanced EGFR mutation-positive non-small cell lung cancer (NSCLC). ${ }^{2}$ Currently, the most commonly used EGFR-TKIs include erlotinib, gefitinib, and icotinib. Although EGFR-TKIs are effective, their adverse effects still exist. Meanwhile, the patient may develop resistance against EGFR-TKIs and the disease may clinically progress after a median treatment of $8-13$ months. For $50 \%-60 \%$ of the resistant patients, resistance develops as T790M mutation emerges. ${ }^{3}$ In 2015 , US Food and Drug Administration approved AZD-9291 for the treatment of EGFR T790M mutation-positive metastatic NSCLC. AZD-9291, also known as osimertinib, is a third-generation EGFR-TKI that selectively inhibits EGFR-TKI-sensitizing and EGFR T790M mutation and brings an obvious efficacy and low incidence of severe side effects. A Phase III trial showed the better curative efficacy and lower adverse event rate of AZD-9291 in the treatment of EGFR mutation-positive advanced NSCLC than standard EGFR-TKIs. ${ }^{4}$ A recent Phase III AURA3 trial demonstrated the superiority of osimertinib over the first-line EGFR-TKI therapy. ${ }^{5}$ 
In this paper, we report a 51-year-old man suffering from extensive epidermal detachment and erosion of mucous membranes, diagnosed with TEN because of the detached area covering $60 \%$ of the whole body surface. The rash occurred 21 days after ingestion of AZD-9291, which is the typical onset. Since no other medicines were taken during this period, AZD-9291 was suspected to be the direct cause of TEN. To the best of our knowledge, no case of TEN induced by AZD-9291 has been described in literature till now.

\section{Case report}

In October 2017, a 51-year-old man, suffering from loose blisters, large-area skin peelings (nearly involving the entire body), mucosal erosion and fever, visited The First Affiliated Hospital with Nanjing Medical University. He had been diagnosed with stage IV EGFR-mutation-positive advanced NSCLC with pleural metastasis in 2016 and received treatments at a thoracic hospital regularly. After chemotherapy (pemetrexed plus cisplatin for six cycles) was completed, icotinib continued to be used till August 2017 when the drug resistance was revealed. Once EGFR T790M mutation was diagnosed, he started taking AZD-9291 with a dose of $80 \mathrm{mg}$ /day to delay the disease progression. Twenty-one days later, he presented with several oral blisters and scattered erythematous maculopapular rashes, which rapidly developed into mucosal erosion and general epidermal detachment involving his entire body with exfoliation on his back skin (Figure 1A and B). When admitted into our department, he was unable to get up owing to severe pain, extreme feebleness, fever, and lower limb edema.

\section{Diagnostic assessment}

The patient was diagnosed with TEN induced by AZD-9291 according to the following parameters:

1. Typical clinical manifestation: Large area of exfoliation involving $>30 \%$ of body surface area, high fever, difficult breathing, and lower limb edema.

2. Laboratory indexes indicating the systemic involvement: Anemia, leukopenia, hypoalbuminemia, hyponatremia.

3. Detailed medical history: No other short-term medication, diet, or exposure before eruption. In addition, the interval between the first administration of AZD-9291 and the disease onset equaled the hypersensitivity response latency.

4. Skin biopsy: Since the above information was indicative enough, biopsy was not performed considering the patient's weak condition.

\section{Therapeutic intervention}

After the patient was enrolled at the hospital, the following measures were taken:

1. AZD-9291 was immediately discontinued.

2. Antiallergic treatment: The patient was treated with intravenous infusion of methylprednisolone ( $40 \mathrm{mg}$, twice per day) combined with immunoglobulin (20 g, once per day for 5 days and then used as appropriate).

3. Symptomatic and supportive treatments: Emergency blood routine and blood chemistry examination showed a low white blood cell count, hypoalbuminemia, and electrolyte disturbance, which prompted us to maintain
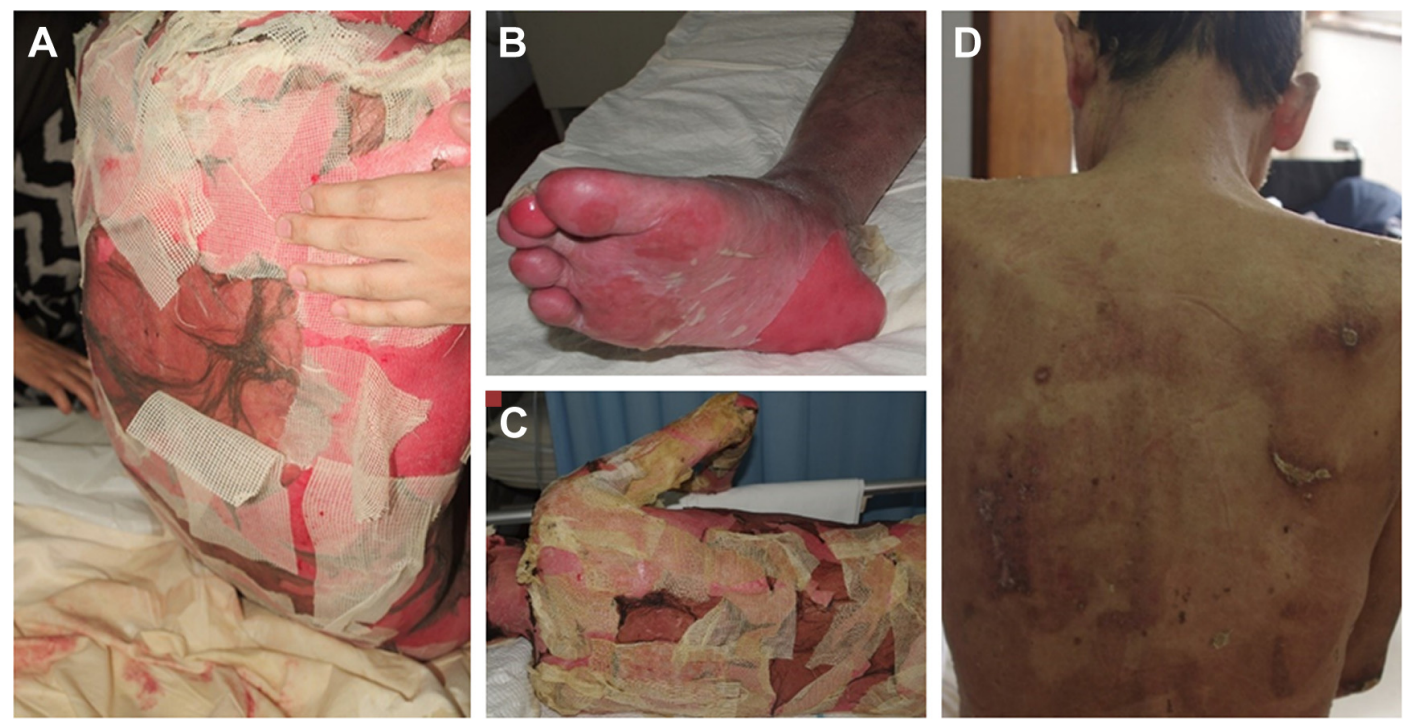

Figure I Skin rash before and after treatment.

Notes: (A) Skin exfoliation on the back. (B) Dermal detachment and loose blisters on the right sole. (C) Back skin after several days of treatment. (D) Skin rash cured after treatment. 
the white blood cell level through supplying albumin and correcting the electrolyte disorder.

4. Daily skin care: Blisters and exudated sites were coated with Ethacridine Zinc Oxide Oil obtained from Institute of Dermatology and Hospital for Skin Diseases, Chinese Academy of Medical Sciences \& Peking Union Medical Collage, Beijing, People's Republic of China once per day. The eroded area was treated with chlorotetracycline ointment and covered with oil gauze once per day. Meanwhile, secretions were regularly collected to detect infection. Erosive genital mucosa was treated with medical biocolloid dispersant several times per day. The blood crusts of ophthalmic, nasal, and oral mucous were cleaned up regularly to prevent conglutination.

5. Monitoring infection closely and timely treatment: On the sixth day after hospitalization, the patient got fever with the body temperature up to $38.3^{\circ} \mathrm{C}$ and the bacterial culture of skin secretions showed Staphylococcus aureus infection. Thereafter, antibiotics (moxifloxacin hydrochloride and sodium chloride injection, intravenous infusion of $400 \mathrm{mg}$ daily) were added and more blood cultures were performed. Several days later, the patient's body temperature went down and skin rashes diminished (Figure 1C), but the tests revealed Acinetobacter baumannii in the sputum and G test and GM test (for testing deep fungal infection but having a low specificity) were positive. According to these results, the antibiotics were upgraded to meropenem (intravenous injection of $0.5 \mathrm{~g}$ every 8 hours), and voriconazole (intravenous infusion of $100 \mathrm{mg}$ twice a day) was infused intravenously to prevent deep fungal infection.

Through these efforts, the patient's rashes healed (Figure 1D) and vital signs became stable 30 days after hospitalization. When discharged from hospital, he could sit on the edge of the bed. Written informed consent was obtained from the patient for publication of this case and the attached images.

\section{Discussion}

SJS/TEN, with an incidence of 1.2-7.4/10,00,000 among adults, ${ }^{6}$ is a rare but painful disease clinically characterized by epidermal exfoliation and systemic symptoms. Inappropriate medication is the main cause of TEN, and high-risk drugs include anti-infective sulfonamides, antiepileptic drugs, nonsteroidal anti-inflammatory oxicam, allopurinol, nevirapine, and chlormezanone. Currently, herbal remedies and new biologicals are also listed as causative agents. ${ }^{7}$
As a disease with genetic predisposition, TEN is more likely to attack patients with particular human leukocyte antigen allotypes. ${ }^{8}$ The pathogenesis of TEN involves antigenic moiety/metabolite, peptide-induced $\mathrm{T}$ cell activation, soluble Fas ligand, perforin/granzyme B, tumor necrosis factor-alpha, nitric oxide, and granulysin. ${ }^{9}$ This complicated pathogenesis makes it hard to standardize the therapeutic strategy for TEN.

The effective treatments for TEN include early diagnosis, immediate withdrawal of suspicious allergenic drugs, symptomatic and supportive treatment. Systematic glucocorticoids combined with immunoglobulin help quickly restrain skin reaction. Intensive skin care is crucially needed for the repair of skin barrier. Meanwhile, efforts should be done to control infection, including closely monitoring infection signs and giving timely treatments.

As the most common cancer worldwide, lung cancer is the leading cause of cancer mortality and has an odds of $80 \%$ to develop NSCLC. ${ }^{10}$ Surgery combined with chemotherapy or radiotherapy is the most effective strategy for NSCLC, but only applicable for the localized tumor. Due to the lack of early clinical manifestation, most patients are usually found at the advanced stage when diagnosed with NSCLC and have lost the golden opportunity of surgery. Before the invention of molecular targeted drugs, metastatic NSCLC was an incurable disease killing victims in a short time. ${ }^{11}$

EGFR is a receptor tyrosine kinase of the ErbB family. When triggered by its potential ligands, EGFR can lead to homo- or heterodimerization of ErbB receptors, then autophosphorylation of the tyrosine domain, and the subsequent signal transduction, such as cell differentiation, proliferation, and apoptosis. In the condition of EGFR deregulation, these signal transduction pathways can be amplified and become uncontrollable, resulting in rapid cell proliferation and antiapoptosis, and even in the development of cancer. ${ }^{11}$ EGFR is obviously overexpressed in NSCLC. NSCLC with EGFR-activated mutations makes up about $10 \%$ of NSCLC cases, ${ }^{12}$ suggesting that EGFR is a potential target for treating NSCLC. EGFR-TKIs are a kind of small molecular inhibitor that specifically functions in the tyrosine domain of EGFR through restraining the activation of tyrosine kinases, binding EGFR and blocking its signaling pathway, and ultimately suppressing tumor cell proliferation and differentiation, and promoting tumor cell apoptosis and other biological reactions. EGFR-TKIs have been approved as an important treatment for NSCLC, especially for NSCLC with EGFR activating mutation. 
Skin rash is the most common side effect of EGFR-TKIs. Since EGFR is highly expressed in skin epithelial cells, the blockade of epidermal growth factor signaling by EGFRTKI will disturb the development of normal epidermis and induce mucocutaneous toxicities, like rash acneiform, skin fissure, and xerosis, which are all symptomatized as pruritus. ${ }^{13}$ To reduce skin rash and drug resistance complicated with the first two generations, the third generation of EGFR-TKIs has been invented. AZD-9291 is a potent, oral, irreversible third-generation EGFR-TKI that inhibits EGFR mutation while sparing wild-type EGFR. ${ }^{11}$ Bearing lower skin toxicity, it is effective for the patients whose drug resistance has mutated T790M.

There are rare reports about severe drug eruption induced by EGFR-TKIs. Huang et al reported TEN was associated with AP and gefitinib combined therapy. ${ }^{8}$ Doesch et al described an EGFR-mutated lung cancer patient developing SJS after the use of afatinib. ${ }^{14}$ AZD-9291 displayed around 200 times greater potency against T790M than wild-type EGFR, resulting in an attractive EGFR-selective agent in comparison with early generation TKIs and less capacity of causing severe skin toxicity. According to our literature review, no serious dermal toxicity caused by AZD-9291 has been reported.

Some scholars have suggested that the severity of the rash is positively correlated with the potency of EGFR-TKIs. A meta-analysis of the association between drug potency and the severity of EGFR-related skin rash revealed that the progression-free survival was longer in patients with skin rash than those without it. ${ }^{15}$ Wacker et al put forward that rash could be used as a new marker for response and possibly be associated with prolonged survival. ${ }^{16}$

When mild or moderate rashes occur, EGFR-TKIs can be continued if rashes are effectively controlled. However, when serious epidermal ablation and necrosis happen, drug must be withdrawn. Accurately assessing the severity of drug reactions and taking the right treatment in time is of great benefit.

Although skin toxicity is a well-known side effect of pemetrexed and there have been some reports of TEN induced by pemetrexed plus cisplatin treatment, ${ }^{17,18}$ we have not suspected pemetrexed plus cisplatin treatment as the cause of this case of TEN, because of the onset time of the disease. TEN usually occurs between 7 and 28 days after the initial drug administration, but the patient successfully completed the chemotherapy for six cycles (21 days every cycle) without skin toxicity, which implies that the chemotherapy has nothing to do with the case of TEN. In addition, the interval between the first administration of AZD-9291 and the disease onset revealed that AZD-9291 was the cause.

The patient of this case took AZD-9291 at a dose of $80 \mathrm{mg} / \mathrm{day}$, and the dose is a recommended dosage. Studies have revealed that doses equivalent to $80 \mathrm{mg}$ could lead to more profound inhibition of tumor growth, and the $80 \mathrm{mg}$ daily dose has been adopted for the optimal dosage based on increasing toxicity at 160 and $240 \mathrm{mg}$ daily combined with similar response rates across all dose levels. The study of Janne et al suggests AZD-9291 has a side-effect profile that is not dose limiting in the majority of patients. ${ }^{19}$ Therefore, the incidence of adverse effects will not increase with the dose addition in the majority of patients. In other words, patients started with a lower dose may not experience lower side effects than the patients with $80 \mathrm{mg}$ daily dose. Because the lower dose possesses lesser efficacy of inhibiting tumor growth and the similar incidence of side effects as the higher dose, we opine that it does not make sense to start taking a lower dose of AZD-9291 and incrementally increase it to control or monitor the side effects.

During the period that this patient discontinued AZD9291 for treating TEN, we surprisingly found that the lung image on the CT scan was clearer than the previous one and the hydrothorax seemed to be less. There is a hypothesis to explain the miraculous improvement that the immune storm of TEN eliminates a part of the cancer cells and suppresses the progression of the cancer. Unfortunately, the improvement of the cancer was transient, and the patient died of respiratory failure 2 months after recovery from TEN. Without anticancer treatment, the growth of cancer cells rapidly took away his life. The Phase II AURA trial has shown the median progression-free survival of AZD-9291 is 11 months. Now, no one can answer the question whether the treatment of AZD-9261 is sufficient in regressing the growth of the NSCLC. Nonetheless, AZD-9291 is proved to be more efficient than the prior EGFR-TKIs and can bring some hope to despairing patients.

We report this case to emphasize the clinical significance of severe rashes after taking EGFR-TKIs. We also hope that more research will be carried out on the association between severe rashes and EGFR-TKIs, and more safe and effective drugs can be developed.

\section{Acknowledgments}

This work was supported by the National Natural Science Foundation of China (81573072) and the Priority Academic Program Development of Jiangsu Higher Education Institutions (JX10231801). 


\section{Disclosure}

The authors report no conflicts of interest in this work.

\section{References}

1. Oakley AM, Krishnamurthy K. Stevens Johnson Syndrome (Toxic Epidermal Necrolysis). Florida: StatPearls Publishing LLC; 2017.

2. Pirker R. What is the best strategy for targeting EGF receptors in nonsmall-cell lung cancer? Future Oncol. 2015;11(1):153-167.

3. Oxnard GR, Arcila ME, Sima CS, et al. Acquired resistance to EGFR tyrosine kinase inhibitors in EGFR-mutant lung cancer: distinct natural history of patients with tumors harboring the T790M mutation. Clin Cancer Res. 2011;17(6):1616-1622.

4. Soria JC, Ohe Y, Vansteenkiste J, et al; FLAURA Investigators. Osimertinib in untreated EGFR-mutated advanced non-small-cell lung cancer. N Engl J Med. 2017;378(2):113-125.

5. Santarpia M, Liguori A, Karachaliou N, et al. Osimertinib in the treatment of non-small-cell lung cancer: design, development and place in therapy. Lung Cancer (Auckl). 2017;8:109-125.

6. Antoon JW, Goldman JL, Lee B, Schwartz A. Incidence, outcomes, and resource use in children with Stevens-Johnson syndrome and toxic epidermal necrolysis. Pediatr Dermatol. 2018;35(2):182-187.

7. Lerch M, Mainetti C, Terziroli Beretta-Piccoli B, Harr T. Current perspectives on Stevens-Johnson syndrome and toxic epidermal necrolysis. Clin Rev Allergy Immunol. 2018;54(1):147-176.

8. Huang JJ, Ma SX, Hou X, et al. Toxic epidermal necrolysis related to AP (pemetrexed plus cisplatin) and gefitinib combination therapy in a patient with metastatic non-small cell lung cancer. Chin J Cancer. 2015; 34(2):94-98.

9. Schwartz RA, McDonough PH, Lee BW. Toxic epidermal necrolysis: part I. Introduction, history, classification, clinical features, systemic manifestations, etiology, and immunopathogenesis. J Am Acad Dermatol. 2013;69(2):171-173.
10. Torre LA, Bray F, Siegel RL, Ferlay J, Lortet-Tieulent J, Jemal A. Global cancer statistics, 2012. CA Cancer J Clin. 2015;65(2):87-108.

11. Lee CC, Shiao HY, Wang WC, Hsieh HP. Small-molecule EGFR tyrosine kinase inhibitors for the treatment of cancer. Expert Opin Investig Drugs. 2014;23(10):1333-1348.

12. Skrzypski M, Szymanowska-Narloch A, Dziadziuszko R. Osimertinibeffective treatment of NSCLC with activating EGFR mutations after progression on EGFR tyrosine kinase inhibitors. Contemp Oncol (Pozn). 2017;21(3):254-258.

13. Kozuki T. Skin problems and EGFR-tyrosine kinase inhibitor. Jpn J Clin Oncol. 2016;46(4):291-298.

14. Doesch J, Debus D, Meyer C, et al. Afatinib-associated Stevens-Johnson syndrome in an EGFR-mutated lung cancer patient. Lung Cancer. 2016; 95:35-38.

15. Liu HB, Wu Y, Lv TF, et al. Skin rash could predict the response to EGFR tyrosine kinase inhibitor and the prognosis for patients with non-small cell lung cancer: a systematic review and meta-analysis. PLoS One. 2013;8(1):e55128.

16. Wacker B, Nagrani T, Weinberg J, Witt K, Clark G, Cagnoni PJ. Correlation between development of rash and efficacy in patients treated with the epidermal growth factor receptor tyrosine kinase inhibitor erlotinib in two large Phase III studies. Clin Cancer Res. 2007;13(13): 3913-3921.

17. Then C, von Einem JC, Muller D, Flaig MJ, Huber RM, Reincke M. Toxic epidermal necrolysis after pemetrexed and cisplatin for non-small cell lung cancer in a patient with sharp syndrome. Onkologie. 2012; 35(12):783-786.

18. Scheinpflug K, Menzel C, Koch A, Kahl C, Achenbach HJ. Toxic epidermal necrolysis related to Cisplatin and pemetrexed for metastatic non-small cell lung cancer. Onkologie. 2012;35(10):600-603.

19. Janne PA, Yang JC, Kim DW, et al. AZD9291 in EGFR inhibitorresistant non-small-cell lung cancer. $N$ Engl J Med. 2015;372(18): 1689-1699.

\section{Publish your work in this journal}

Drug Design, Development and Therapy is an international, peerreviewed open-access journal that spans the spectrum of drug design and development through to clinical applications. Clinical outcomes, patient safety, and programs for the development and effective, safe, and sustained use of medicines are the features of the journal, which

\section{Dovepress}

has also been accepted for indexing on PubMed Central. The manuscript management system is completely online and includes a very quick and fair peer-review system, which is all easy to use. Visit http://www.dovepress.com/testimonials.php to read real quotes from published authors. 